\title{
On a Limit Theorem for Branching One- Dimensional Diffusion Processes
}

\author{
Dedicated to Professor $K$. Itô on his 60th birthday \\ By \\ Seiji KageYAMA* and Yukio OguRA**
}

\section{§1. Introduction}

Let $M$ be a simply connected complete Riemannian manifold with constant sectional curvature, and consider a branching Brownian motion $Y=\left(y_{t}, \mathbb{P}_{y}\right)$ having $M$ as the underlying state space ([5]). In the case of $M=S^{d}$, the $d$ dimensional sphere, one can apply the results of Watanabe [16], [17] and Asmussen-Hering [1] to obtain a limit theorem on the number of particles in a domain for the process $\boldsymbol{Y}$. If $M=\mathbb{R}^{d}$, then, although $M$ is not compact, the process $\boldsymbol{Y}$ belongs to the class considered by Watanabe [17], and his argument works well. But, if $M$ has constant negative sectional curvature $-k$, then $Y$ is not necessarily contained in the scheme of [17] and a new phenomenon appears. In this case, the Laplace-Beltrami operator is given by

$$
\Delta=\partial^{2} / \partial r^{2}+\sqrt{k}(d-1) \operatorname{coth} \sqrt{k} r \partial / \partial r+\Delta^{\prime}
$$

in polar coordinates, where $\Delta^{\prime}$ is the Laplace-Beltrami operator on the sphere $S_{r}=\{y \in M$ : distance $(y, 0)=r\} \quad([4]$ p. 445$)$. Hence the radial part process $X$ of the branching Brownian motion $\boldsymbol{Y}$ on $M$ is reduced to a branching diffusion process on the underlying state space $S=[0, \infty)$, whose nonbranching part diffusion has the generator

$$
L=d^{2} / 2 d r^{2}+\sqrt{k}(d-1) / 2 \operatorname{coth} \sqrt{k} r d / d r .
$$

The spectrum of $L$ is the interval $\left(-\infty,-k(d-1)^{2} / 8\right]$ and there exist many

Communicated by K. Itô, May 25, 1978.

* Tachibana High School.

** Department of Mathematics, Saga University. The second author was supported by Kagakukenkyuhi-Hojokin 254039. 
bounded nonnegative martingales for $\boldsymbol{X}$ (Section 5). This enables us to show that bounded domains may come to contain no particle in some cases, even when the total number of particles diverges to infinity as time elapses.

Having the above situation in the background, we shall study in this paper the branching diffusion process on the underlying state space $S=[0, \infty)$, whose nonbranching part diffusion has the generator

$$
L=d^{2} / 2 d x^{2}+b(x) d / d x .
$$

Precise conditions on $b(x)$ as well as boundary conditions will be given in Section 2. The radial part process $\mathbb{X}$ mentioned above is contained in our class. Our results are fully described in Section 2. We shall prepare some lemmas on ordinary differential equations in Section 3 and some comparison theorems for stochastic differential equations in Section 4. Sections 5 and 6 are devoted to the proof of Theorems.

The authors would like to express their sincere gratitude to Professor N. Ikeda, who drew their interest to this subject. They would also like to thank the referee for correcting some errors and improving a lot of expressions.

\section{§2. Notations and Results}

Throughout this paper, we assume that $b(x)$ is a function satisfying the following conditions:

(2.1) $b(x)$ is defined on $S=[0, \infty)$ and $b(0)=0$,

(2.2) $b(x)$ is continuous and nonincreasing on $(0, \infty)$,

(2.3) $b(x) \geqq b_{0}>0$ on $(0, \infty)$,

(2.4) $\int_{R}^{\infty} x\left(b(x)-b_{0}\right) d x<\infty \quad$ for every $R>0$.

Let

$$
s(x)=\int_{1}^{x} e^{-B(y)} d y, \quad x \in S, \quad m(E)=2 \int_{E} e^{B(x)} d x, \quad E \in \mathscr{B}(S),
$$

where $B(x)=2 \int_{1}^{x} b(y) d y$ and $\mathscr{B}(S)$ is the topological Borel field of $S$. Then there exists a unique conservative diffusion process $X=\left(x_{t}, P_{x}\right)$ on $[0, \infty)$ with scale $s(x)$ and speed measure $m(d x)$ satisfying

$$
P_{0}\left(\sigma_{0+}=0\right)=1^{1)}
$$

1) $\sigma_{x}$ is the first hitting time of $x_{t}$ for the point $x$, and $\sigma_{0+}=\lim _{x \downarrow 0} \sigma_{x}$. 
([8]). The boundary $\infty$ is natural in Feller's sense (ibid. p. 108). Further, since $X$ is conservative and $m(\{0\})=0,(2.3)$ and (2.5) ensure that the boundary 0 is reflecting if

$$
e^{-B(x)} \int_{x}^{1} 2 e^{B(y)} d y \in L^{1}(0,1) .
$$

The boundary 0 is entrance non-exit if

$$
e^{-B(x)} \int_{x}^{1} 2 e^{B(y)} d y \notin L^{1}(0,1)
$$

(ibid.). The generator of $X$ coincides with $L$ of (1.2) with the domain

$$
D(L)=\left\{u \in \mathbb{C}(S): L u \in \mathbb{C}(S), \lim _{x \downarrow 0} e^{B(x)} u^{\prime}(x)=0\right\}
$$

(ibid.). Note that the last relation in the braces of (2.6) is automatically satisfied in the case of (E). We denote the transition density for the diffusion $X$ with respect to $m(d x)$ by $p(t, x, y)$, and the semigroup by $T_{t}$.

Following [5], let $S^{n}$ be the $n$-fold symmetric product of $S\left(S^{0}=\partial, \partial\right.$ is an extra point), and set $S=\bigcup_{n=0}^{\infty} S^{n}$. Thus an element $x \in S$ belongs to some $S^{n}$, and, if $n \geqq 1$, we have a coordinate expression $\boldsymbol{x}=\left[x^{1}, x^{2}, \ldots, x^{n}\right]$. Define a stochastic kernel $\pi(x, \mathbb{E})(x \in S, \boldsymbol{E} \in \mathscr{B}(S))$ by $\pi(x, \mathbb{E})=1$ if $[x, x] \in \mathbb{E}$, and $=0$ otherwise. Then, for a positive constant $c$, there exists a unique branching diffusion process (BDP) $\boldsymbol{X}=\left(\boldsymbol{x}_{t}, \boldsymbol{P}_{\lambda}\right)(x \in S)$ on $S$ corresponding to the fundamental system $(X, c, \pi) . \quad X$ is called the nonbranching part, $c$ the branching rate and $\pi$ the branching law.

Let $\boldsymbol{B}$ be the set of all bounded Borel measurable functions on $S$. For each $g \in \boldsymbol{B}$, set

$$
\begin{aligned}
& \hat{g}(\boldsymbol{x})= \begin{cases}g\left(x^{1}\right) g\left(x^{2}\right) \cdots g\left(x^{n}\right), & \boldsymbol{x}=\left[x^{1}, x^{2}, \ldots, x^{n}\right] \in \boldsymbol{S} \backslash\{\partial\}, \\
1, & \boldsymbol{x}=\partial,\end{cases} \\
& \check{g}(\boldsymbol{x})= \begin{cases}g\left(x^{1}\right)+g\left(x^{2}\right)+\cdots+g\left(x^{n}\right), & \boldsymbol{x}=\left[x^{1}, x^{2}, \ldots, x^{n}\right] \in \boldsymbol{S} \backslash\{\partial\}, \\
0, & \boldsymbol{x}=\partial .\end{cases}
\end{aligned}
$$

Then, for each continuous $g \in \boldsymbol{B}$ with $\|g\| \leqq 1$, the function $u(t, x)=\mathbb{E}_{x}\left[\hat{g}\left(\boldsymbol{x}_{t}\right)\right]$ satisfies the so-called $S$-equation

$$
\frac{\partial u}{\partial t}=L u+c\left(u^{2}-u\right), \quad u(0+, x)=g(x)^{2)}
$$

(cf. [5]). Let, for $\alpha, l \in \boldsymbol{R}$,

2) More precisely, $u(t, \cdot) \in D(L)$ and (2.7) is satisfied. In writing similar equations, the condition that $u$ belongs to $D(L)$ is meant implicitly. 


$$
\begin{aligned}
& \boldsymbol{B}_{i}(\alpha, l)=\left\{g \in \boldsymbol{B}: \lim _{x \rightarrow \infty} e^{\alpha x} g(x) / x^{i}=l\right\}, \\
& \boldsymbol{B}_{i}(\alpha)=\bigcup_{l \in \boldsymbol{R}} \boldsymbol{B}_{i}(\alpha, l), \quad i=0,1 .
\end{aligned}
$$

Thus, each $g \in \boldsymbol{B}_{i}(\alpha)$ belongs to $\boldsymbol{B}_{i}(\alpha, l)$ for some $l \in \boldsymbol{R}$, which we denote by $l(g)$.

Finally, set

$$
\lambda_{0}=b_{0}^{2} / 2, \quad \alpha_{\lambda}=b_{0}-\sqrt{b_{0}^{2}-2 \lambda}, \quad \alpha_{\lambda_{0}}=b_{0} .
$$

Now we are ready to state our results.

Theorem 1. 1) For each $0 \leqq \lambda<\lambda_{0}$, there is a nonnegative integrable random variable $W_{\lambda}$ such that for each $g \in \boldsymbol{B}_{0}\left(\alpha_{\lambda}, l\right)$

$$
\lim _{t \rightarrow \infty} e^{(\lambda-c) t} \check{g}\left(x_{t}\right)=l W_{\lambda}, \quad \text { a.s. }
$$

2) There is a nonnegative integrable random variable $W=W_{\lambda_{0}}$ such that for each $g \in \boldsymbol{B}_{1}\left(\alpha_{\lambda_{0}}, l\right)$

$$
\lim _{t \rightarrow \infty} e^{\left(\lambda_{0}-c\right) t} \check{g}\left(x_{t}\right)=l W, \quad \text { in probability. }
$$

Theorem 2. If $0 \leqq \lambda \leqq \lambda_{0}$ and $\alpha_{\lambda}^{2} \leqq 2 c$, then

$$
\boldsymbol{P}_{x}\left(0<W_{\lambda}<\infty\right)=1, \quad x>0 .
$$

Remark 1. Consider the case $0<c<\lambda_{0}\left[c=\lambda_{0}\right]$. Theorem 1 says that

$$
\lim _{x \rightarrow \infty} e^{\alpha_{c} x} g(x)=l \quad\left[\text { resp. } \lim _{x \rightarrow \infty} e^{b_{0} x} g(x) / x=l\right]
$$

implies

$$
\lim _{t \rightarrow \infty} \breve{g}\left(x_{t}\right)=l W_{\lambda}, \text { a.s. } \quad[=l W, \text { in probability }] \text {. }
$$

Further, Theorems 1 and 2 say that

$$
\lim _{x \rightarrow \infty} e^{\alpha_{c} x} g(x)=\infty \quad\left[\text { resp. } \lim _{x \rightarrow \infty} e^{b_{0} x} g(x) / x=\infty\right]
$$

implies

$$
\lim _{t \rightarrow \infty} \breve{g}\left(x_{t}\right)=\infty, \quad \text { a.s. [resp. in probability]. }
$$

Indeed, for $0 \leqq \lambda<\lambda_{0}\left[\lambda=\lambda_{0}\right], \alpha_{\lambda}$ is the smaller [resp. unique] solution of

$$
t^{2}-2 b_{0} t+2 \lambda=0 \text {. }
$$

Hence $\alpha_{\lambda}^{2} \leqq 2 \lambda$, and (2.10) holds for $\lambda=c$. 


\section{§3. Lemmas on Ordinary Differential Equations}

Lemma 1. 1) For each $\lambda$, there is a unique solution $\varphi_{\lambda}(x)$ of the equation

$$
L \varphi_{\lambda}(x)=-\lambda \varphi_{\lambda}(x), \quad \varphi_{\lambda}(0)=1 .
$$

2) If $0<\lambda \leqq \lambda_{0}$, then $\varphi_{\lambda}(x)$ is positive and decreasing. $\varphi_{0}(x) \equiv 1$. Further,

$$
\begin{array}{ll}
\varphi_{\lambda} \in \boldsymbol{B}_{0}\left(\alpha_{\lambda}\right), & 0 \leqq \lambda<\lambda_{0}, \\
\varphi_{\lambda_{0}} \in \boldsymbol{B}_{1}\left(b_{0}\right), & \lambda=\lambda_{0}
\end{array}
$$

with positive $l\left(\varphi_{\lambda}\right)$.

3) If $\lambda>\lambda_{0}$, then $\varphi_{\lambda}(x)<0$ for some $x \in(0, \infty)$.

Proof. 1) First we shall show the uniqueness. Let $\varphi_{\lambda}(x)$ be a solution of (3.1), and put $\xi(x)=\varphi_{\lambda}(x)$ and $\eta(x)=\varphi_{\lambda}^{\prime}(x)^{3)}$. Then (3.1) is equivalent to

$$
\left\{\begin{array}{l}
\xi^{\prime}(x)=\eta(x), \\
\eta^{\prime}(x)=-2 \lambda \xi(x)-2 b(x) \eta(x), \quad \xi(0)=1
\end{array}\right.
$$

and

$$
\lim _{x \downarrow 0} e^{B(x)} \eta(x)=0 .
$$

Solving the second equation of (3.4), we have

$$
\eta(x)=-2 \lambda \int_{x_{1}}^{x} \xi(y) e^{-2 \int_{y}^{x} b(z) d z} d y+e^{-B(x)} e^{B\left(x_{1}\right)} \eta\left(x_{1}\right),
$$

for $x, x_{1} \in(0, \infty)$. Hence, by letting $x_{1} \downarrow 0$, we get

$$
\eta(x)=-2 \lambda \int_{0}^{x} \xi(y) e^{-2 \int_{y}^{x} b(z) d z} d y
$$

This and (3.4) give

$$
\xi(x)=1-2 \lambda \int_{0}^{x} \int_{0}^{y} \xi(z) e^{-2 \int_{z}^{y} b(u) d u} d z d y .
$$

Hence the solution $\xi(x)$ of $(3.7)$ is unique. This means the uniqueness of the solution $\varphi_{\lambda}(x)$ of (3.1).

The existence of the solution $\varphi_{\lambda}(x)$ of (3.1) is obvious, because it is easy to see that (3.7) has a solution $\xi(x)$.

2) Clearly, $\varphi_{0}(x) \equiv 1$. We divide the proof for the case $0<\lambda \leqq \lambda_{0}$ into two steps.

3) $\varphi_{\lambda}^{\prime}(x)=d \varphi_{\lambda}(x) / d x$. 
Step 1. We shall show that $\varphi_{\lambda}(x)$ is positive and decreasing. It is enough to see that $\xi(x)=\varphi_{\lambda}(x)>0$ and $\eta(x)=\varphi_{\lambda}^{\prime}(x)<0$.

By (3.6) and (3.7),

$$
\xi(x)>0, \quad \eta(x)<0, \quad 0<x<\delta,
$$

for some $\delta>0$. Set $T=\inf \{x>0: \xi(x)=0\}^{4)}$ and $\zeta(x)=-\eta(x) / \xi(x), x \in(0, T)$. Then $\zeta(0+)=0$ and $\zeta(x)>0$ for $0<x<\delta$. Further, by (3.4),

$$
\zeta^{\prime}(x)=\zeta(x)^{2}-2 b(x) \zeta(x)+2 \lambda, \quad x \in(0, T) .
$$

This implies

$$
\zeta(x)>0, \quad x \in(0, T) .
$$

Indeed, if otherwise, contradiction occurs at the first zero point $x_{0} \in(0, T)$ of $\zeta(x)$, because $\zeta^{\prime}\left(x_{0}\right) \leqq 0$ whereas $\zeta^{\prime}\left(x_{0}\right)=2 \lambda>0$.

Now (3.9) and (3.10) yield

$$
\zeta^{\prime}(x) \leqq \zeta(x)^{2}-2 b_{0} \zeta(x)+2 \lambda, \quad x \in(0, T) .
$$

Define $\alpha_{+}$and $\alpha_{-}$by

$$
\alpha_{ \pm} \equiv \alpha_{ \pm}(\lambda)=b_{0} \pm \sqrt{b_{0}^{2}-2 \lambda} .
$$

Note that $\alpha_{-}=\alpha_{\lambda}$ and that (3.11) implies

$$
\zeta^{\prime}(x) \leqq\left(\zeta(x)-\alpha_{+}\right)\left(\zeta(x)-\alpha_{-}\right), \quad x \in(0, T) .
$$

If $0<\lambda<\lambda_{0}$, then this yields

$$
0<\zeta(x)<\alpha_{-}, \quad x \in(0, T) .
$$

If $\lambda=\lambda_{0}$, then (3.12) is written as $\zeta^{\prime}(x) \leqq\left(\zeta(x)-b_{0}\right)^{2}$. On the other hand, there is a $\delta_{0} \in(0, T)$ such that $0<\zeta(x)<b_{0}$ for $x \in\left(0, \delta_{0}\right]$. Take any $x_{1} \in\left(\delta_{0}, T\right)$ and fix it. If $\zeta(x)<b_{0}$ for $\delta_{0}<x<x_{1}$, then

$$
\frac{1}{b_{0}-\zeta(x)} \leqq x-\delta_{0}+\frac{1}{b_{0}-\zeta\left(\delta_{0}\right)} .
$$

This excludes the possibility that $\zeta(x) \uparrow b_{0}$ as $x \uparrow x_{1}$. Hence (3.13) holds in this case also.

Now we shall show $T=\infty$. Suppose $T<\infty$. Then $\eta(T)=0$ by (3.13). Since the solution of (3.4) for $x \geqq T$ with the initial condition $\xi(T)=\eta(T)=0$ is unique, we have $\xi(x)=\eta(x)=0$ for $x \geqq T$. Then, by (3.7),

4) We always consider that the infimum of the empty set is $\infty$. 


$$
0=\xi(x)=\xi(T)-2 \lambda \int_{T}^{x} \int_{0}^{y} \xi(z) e^{-2 \int_{z}^{y} b(u) d u} d z d y<0, \quad x>T .
$$

This is a contradiction.

Step 2. We shall show the second assertion. By (3.4),

$$
\left(\begin{array}{c}
\dot{\zeta}^{\prime} \\
\eta^{\prime}
\end{array}\right)=A\left(\begin{array}{l}
\xi \\
\eta
\end{array}\right)-\left(\begin{array}{c}
0 \\
2 \tilde{b} \eta
\end{array}\right),
$$

where $A=\left(\begin{array}{cc}0 & 1 \\ -2 \lambda & -2 b_{0}\end{array}\right)$ and $\tilde{b}(x)=b(x)-b_{0} . \quad$ It follows that, for each $x_{0}>0$,

(3.14) $\left(\begin{array}{c}\xi(x) \\ \eta(x)\end{array}\right)=-\int_{x_{0}}^{x} e^{(x-y) A}\left(\begin{array}{c}0 \\ 2 \tilde{b}(y) \eta(y)\end{array}\right) d y+e^{\left(x-x_{0}\right) A}\left(\begin{array}{c}\xi\left(x_{0}\right) \\ \eta\left(x_{0}\right)\end{array}\right)$.

Now suppose that $0<\lambda<\lambda_{0}$. Then

$$
e^{x A}=\frac{1}{\alpha_{+}-\alpha_{-}}\left(\begin{array}{ll}
\alpha_{+} e^{-\alpha_{-} x}-\alpha_{-} e^{-\alpha_{+} x} & e^{-\alpha_{-} x}-e^{-\alpha_{+} x} \\
2 \lambda\left(e^{-\alpha_{+} x}-e^{-\alpha_{-} x}\right) & \alpha_{+} e^{-\alpha_{+} x}-\alpha_{-} e^{-\alpha_{-} x}
\end{array}\right) .
$$

Hence, noting $\xi(0)=1$ and $\eta(0+)=0$ (by (3.6)), we have

$$
\begin{aligned}
\xi(x)=\frac{1}{\alpha_{+}-\alpha_{-}}\left(\alpha_{+} e^{-\alpha_{-} x}-\alpha_{-} e^{-\alpha_{+} x}\right. & \\
& \left.\quad-\int_{0}^{x}\left(e^{-\alpha_{-}(x-y)}-e^{-\alpha_{+}(x-y)}\right) 2 \tilde{b}(y) \eta(y) d y\right),
\end{aligned}
$$

as well as convergence of the integral. On the other hand, (3.14) and (3.15) imply

$$
e^{\alpha-x}|\eta(x)| \leqq K_{1} \int_{x_{0}}^{x} \tilde{b}(y) e^{\alpha-y}|\eta(y)| d y+K_{2}
$$

for some $K_{1}, K_{2}>0$. Hence, by Gronwall's inequality and (2.4), we have

$$
e^{\alpha-x}|\eta(x)| \leqq K_{2} \exp \left\{K_{1} \int_{x_{0}}^{x} \tilde{b}(y) d y\right\} \leqq K_{3}<\infty, x>x_{0},
$$

for some $K_{3}>0$. Now, using this and (2.4) again, we obtain (3.2) with $l\left(\varphi_{\lambda}\right)>0$ from (3.16).

Turning to the case of $\lambda=\lambda_{0}$, we have

$$
e^{x A}=e^{-b_{0} x}\left(\begin{array}{cc}
1+b_{0} x & x \\
-4 b_{0}^{2} x & 1-b_{0} x
\end{array}\right) .
$$

Hence (3.14) gives 


$$
\begin{gathered}
\xi(x)=x e^{-b_{0} x}\left(b_{0}-\int_{0}^{x} e^{b_{0} y}\left(1-\frac{y}{x}\right) 2 \tilde{b}(y) \eta(y) d y\right)+e^{-b_{0} x} \\
e^{b_{0} x}|\eta(x)| / x \leqq K_{5} \int_{x_{0}}^{x} y \tilde{b}(y) \frac{e^{b_{0} y}|\eta(y)|}{y} d y+K_{6} .
\end{gathered}
$$

By Gronwall's inequality, it follows from (3.19) and (2.4) that

$$
e^{b_{0} x}|\eta(x)| / x \leqq K_{6} \exp \left\{K_{5} \int_{x_{0}}^{x} y \tilde{b}(y) d y\right\} \leqq K_{7}<\infty .
$$

Hence we have (3.3) with $l\left(\varphi_{\lambda_{0}}\right)>0$ from (3.18) and (2.4).

3) Let $\lambda>\lambda_{0}$. Let $T$ and $\zeta(x)$ be as in 2) Step 1. First we shall show $T<\infty$. Suppose $T=\infty$, and choose an $\varepsilon>0$ such that $2 \lambda-\left(b_{0}+\varepsilon\right)^{2}=a^{2}$ for some $a>0$. Then there is an $x_{0}>0$ satisfying

$$
b(x) \leqq b_{0}+\varepsilon, \quad x \geqq x_{0} .
$$

We note that (3.9) and (3.10) are valid also in this case. Hence,

$$
\zeta^{\prime}(x) \geqq\left(\zeta(x)-b_{0}-\varepsilon\right)^{2}+a^{2}, \quad x \geqq x_{0} .
$$

Solving this, we have

$$
\operatorname{Tan}^{-1}\left[\left(\zeta(x)-b_{0}-\varepsilon\right) / a\right]-\operatorname{Tan}^{-1}\left[\left(\zeta\left(x_{0}\right)-b_{0}-\varepsilon\right) / a\right] \geqq a\left(x-x_{0}\right), \quad x \geqq x_{0},
$$

which is absurd. Hence $T<\infty$. If $\eta(T)=0$, then $\xi(T)=\eta(T)=0$. This is impossible by the last argument in 2) Step 1 . Thus $\eta(T)<0$ since $\zeta(x)>0$ on $(0, T)$. This implies $\varphi_{\lambda}(x)=\xi(x)<0$ for some $x>T$.

q.e.d.

Remark 2. The spectrum of $L$ on the space $L^{2}(m(d x))$ is contained in $\left(-\infty,-\lambda_{0}\right]$. Further, $-\lambda_{0}$ belongs to the spectrum of $L$.

Proof. Lemma 1 implies

$$
T_{t} g(x)=O\left(e^{-\lambda_{0} t}\right), \quad t \rightarrow \infty,
$$

for each $g \in \boldsymbol{B}$ with compact support. This assures the first assertion.

If $-\lambda_{0}$ does not belong to the spectrum of $L$, then the spectrum is contained in $\left(-\infty,-\lambda_{0}-\delta\right]$ for some $\delta>0$, because it is closed. Hence $p(t, x, y)$ $=O\left(e^{-\left(\lambda_{0}+\delta\right) t}\right)$ as $t \rightarrow \infty$, and the Green kernel

$$
G_{\lambda}(x, y)=\int_{0}^{\infty} e^{\lambda t} p(t, x, y) d t
$$

is convergent for $\lambda_{0}<\lambda<\lambda_{0}+\delta / 2$. This, combined with Dynkin's formula, yields $E_{0+}\left[e^{\lambda \sigma_{x}}\right]<\infty$. Hence $\tilde{\varphi}_{\lambda}(x) \equiv 1 / E_{0+}\left[e^{\lambda \sigma_{x}}\right]$ is positive. But $\tilde{\varphi}_{\lambda}(x)$ solves (3.1) (cf. [8] 4.6), which contradicts Lemma 1. 
Lemma 2. 1) Let $0<c \leqq \lambda_{0}$. Then, for each $0 \leqq v_{0} \leqq 1$, there is a unique solution of the equation

$$
\left\{\begin{array}{l}
L v+c\left(v^{2}-v\right)=0 \\
v(0)=v_{0}, \quad 0 \leqq v \leqq 1
\end{array}\right.
$$

Further, if $0<v_{0}<1$,

$$
\begin{array}{ll}
1-v(x) \in \boldsymbol{B}_{0}\left(\alpha_{c}\right), & 0<c<\lambda_{0}, \\
1-v(x) \in \boldsymbol{B}_{1}\left(b_{0}\right), & c=\lambda_{0}
\end{array}
$$

with positive $l(1-v)$.

2) If $c>\lambda_{0}$ and $0<v_{0}<1$, then (3.20) admits no solution.

Proof. The outline of the proof is similar to that of Lemma 1.

1) Step 1 (Uniqueness). Set $\xi(x)=1-v(x), \eta(x)=-v^{\prime}(x)$, and

$$
f(\xi)= \begin{cases}2 c \xi(1-\xi), & 0 \leqq \xi \leqq 1 \\ 0, & \text { otherwise. }\end{cases}
$$

Then (3.20) is rewritten as

$$
\left\{\begin{array}{l}
\xi^{\prime}(x)=\eta(x), \\
\eta^{\prime}(x)=-f(\xi(x))-2 b(x) \eta(x), \\
\xi(0)=1-v_{0}, \quad 0 \leqq \xi(x) \leqq 1
\end{array}\right.
$$

with (3.5). Then, as in the proof of Lemma 1, we have

$$
\begin{gathered}
\eta(x)=-\int_{0}^{x} f(\xi(y)) e^{-2 \int_{y}^{x} b(z) d z} d y \\
\xi(x)=1-v_{0}-\int_{0}^{x} \int_{0}^{y} f(\xi(z)) e^{-2 \int_{z}^{y} b(u) d u} d z d y .
\end{gathered}
$$

Now the uniqueness follows, since $f(\xi)$ is Lipschitz continuous.

Step 2 (Existence). Since there is a trivial solution $v(x) \equiv 0$ (resp. $\equiv 1$ ) in the case of $v_{0}=0$ (resp. $=1$ ), we assume $0<v_{0}<1$. Further, (3.25) has a unique solution $\xi(x)$. So it is enough to show

$$
0<\xi(x)<1, \quad x>0 .
$$

By (3.24) and (3.25), there exists a positive $\delta$ such that

$$
0<\xi(x)<1, \quad \eta(x)<0, \quad 0<x<\delta .
$$

Set $T=\inf \{x>0: \xi(x)(1-\xi(x))=0\}$ and $\zeta(x)=-\eta(x) / \xi(x)$ for $x \in(0, T)$. Then (3.23) gives 


$$
\zeta^{\prime}(x)=\zeta(x)^{2}-2 b(x) \zeta(x)+2 c(1-\xi(x)), \quad x \in(0, T) .
$$

It follows from this and (3.27) that $\zeta(x)>0$ for $x \in(0, T)$. Hence,

$$
\zeta^{\prime}(x) \leqq\left(\zeta(x)-\alpha_{+}\right)\left(\zeta(x)-\alpha_{-}\right), \quad x \in(0, T),
$$

where

$$
\alpha_{ \pm}=\alpha_{ \pm}(c)=b_{0} \pm \sqrt{b_{0}^{2}-2 c} .
$$

Now, as in the proof of Lemma 1 , we have

$$
0<\zeta(x)<\alpha_{-}, \quad x \in(0, T) .
$$

Suppose that $T<\infty$. Then, since $\xi^{\prime}(x)=\eta(x)<0$ for $x \in(0, T)$, it must hold that $\xi(T)=\eta(T)=0$. But this leads to contradiction by the same argument as in the proof of Lemma 1 . Hence $T=\infty$.

Step 3 (Proof of the second assertion). First we shall show

$$
\lim _{x \rightarrow \infty} \xi(x)=0 .
$$

Since $\xi(x)$ is decreasing, it tends to a limit $\xi_{*}$. If $\xi_{*} \neq 0$, then, by (3.24),

$$
\varlimsup_{x \rightarrow \infty} \eta(x) \leqq-e^{-2 \delta b_{0}} \lim _{x \rightarrow \infty} \int_{x-\delta}^{x} f(\xi(y)) e^{-2 \int_{y}^{x} \tilde{b}(z) d z} d y=-\delta e^{-2 \delta b_{0}} f\left(\xi_{*}\right)<0 .
$$

This contradicts the fact $\xi(x) \geqq 0, x>0$. Hence, $\xi_{*}=0$.

Now, since $\eta(0+)=0,(3.23)$ is written as

$$
\left(\begin{array}{l}
\xi^{\prime} \\
\eta^{\prime}
\end{array}\right)=A\left(\begin{array}{l}
\xi \\
\eta
\end{array}\right)+\left(\begin{array}{l}
0 \\
\gamma
\end{array}\right),\left(\begin{array}{l}
\xi(0+) \\
\eta(0+)
\end{array}\right)=\left(\begin{array}{c}
1-v_{0} \\
0
\end{array}\right),
$$

where $A=\left(\begin{array}{cc}0 & 1 \\ -2 c & -2 b_{0}\end{array}\right)$ and

$$
\gamma=\gamma(x)=2 c \xi(x)^{2}-2 \tilde{b}(x) \eta(x) .
$$

Let $0<c<\lambda_{0}$. Then as in the proof of Lemma 1 , we have

$$
\begin{aligned}
\xi(x)=\frac{1}{\alpha_{+}-\alpha_{-}}\left[\int_{0}^{x}\left(e^{-\alpha_{-}(x-y)}-e^{-\alpha+(x-y)}\right) \gamma(y) d y\right. & \\
& \left.+\left(\alpha_{+} e^{-\alpha_{-} x}-\alpha_{-} e^{-\alpha_{+} x}\right)\left(1-v_{0}\right)\right]
\end{aligned}
$$

and, for each $x_{0}>0$,

$$
|\eta(x)| \leqq K_{1} \int_{x_{0}}^{x} e^{-\alpha-(x-y)} \gamma(y)|\eta(y)| d y+K_{2} e^{-\alpha-x} .
$$


Let $\mu(x)=\xi(x)+|\eta(x)|$. Then (3.33)-(3.35) imply

$$
e^{\alpha-x} \mu(x) \leqq K_{3} \int_{x_{0}}^{x}(\xi(y)+\tilde{b}(y)) e^{\alpha-y} \mu(y) d y+K_{4}, \quad x \geqq x_{0} .
$$

Hence, by Gronwall's inequality,

$$
e^{\alpha-x} \mu(x) \leqq K_{4} \exp \left\{K_{3} \int_{x_{0}}^{x}(\tilde{b}(y)+\xi(y)) d y\right\} .
$$

By (3.31) and by $\lim _{x \rightarrow \infty} \tilde{b}(x)=0$, this gives

$$
\mu(x)=O\left(e^{-\left(\alpha_{-}-\delta\right) x}\right), \quad x \rightarrow \infty
$$

for each $\delta>0$. Since $\xi(x) \leqq \mu(x),(3.36)$ combined with (3.37) and (2.4) assures boundedness of $e^{-\alpha_{-} x} \mu(x)$. Hence (3.21) and the inequality $l(1-v)>0$ follow from (3.34), (3.33) and (2.4).

In case $c=\lambda_{0}$, we have

$$
\begin{gathered}
\xi(x)=\int_{0}^{x} e^{-b_{0}(x-y)}(x-y) \gamma(y) d y+\left(1+b_{0} x\right) e^{-b_{0} x}\left(1-v_{0}\right), \\
|\eta(x)| \leqq K_{5} \int_{x_{0}}^{x} e^{-b_{0}(x-y)}(x-y) \gamma(y) d y+K_{6} x e^{-b_{0} x}
\end{gathered}
$$

in place of (3.34) and (3.35). Hence, as above,

$$
\frac{e^{b_{0} x} \mu(x)}{x} \leqq K_{7} \int_{x_{0}}^{x}(y \xi(y)+y \tilde{b}(y)) \frac{e^{b_{0} y} \mu(y)}{y} d y+K_{8}, \quad x \geqq x_{0} .
$$

This implies (3.37) first, and then boundedness of $e^{b_{0} x} \mu(x) / x$. Now (3.22) and the inequality $l(1-v)>0$ follow from (3.38), (3.33) and (2.4).

2) Suppose that $c>\lambda_{0}, 0<v_{0}<1$, and $v(x)$ satisfies (3.20) except for $0 \leqq$ $v(x) \leqq 1$. Notice that the argument in 1) Step 1 is valid also in this case. Thus (3.27) holds. Use $T$ and $\zeta(x)$ in 1) Step 2. Then (3.28) follows. Now we shall show $T<\infty$. Suppose that $T=\infty$. Then we have (3.31) by the previous argument. Choose an $\varepsilon>0$ satisfying $2 c(1-\varepsilon)-\left(b_{0}+\varepsilon\right)^{2}>0$. We can find an $x_{0}$ $>0$ such that $\xi(x)<\varepsilon$ and $b(x) \leqq b_{0}+\varepsilon$ for $x \geqq x_{0}$. This and (3.28) lead to a contradiction in the same way as in the proof of Lemma 1. Hence $T<\infty$. Since $\eta(x)<0$ on $(0, T), \xi(T)$ must be 0 . Thus it is enough to. show $\eta(T)<0$. But this is clear if we repeat the consideration in the proof of Lemma 1 . q.e.d.

\section{§4. Comparison Theorem for Stochastic Differential Equations}

A comparison theorem for one-dimensional stochastic differential equations 
is found in [6] and [18]. Here we shall reformulate it in a form convenient for our use.

Let $\left(\beta(t), \mathscr{F}_{t}, P\right)^{5)}$ be a one-dimensional Brownian motion and consider a stochastic integral equation

$$
x(t)=x_{0}+\beta(t)+\int_{0}^{t} b(x(s)) d s+\psi(t), \quad x_{0}>0 .
$$

We say that $(x(t), \psi(t))$ is a solution of (4.1), if $x(t)$ and $\psi(t)$ are continuous and $\mathscr{F}_{t}$-adapted, $x(t)$ is nonnegative, $\psi(t)$ is nondecreasing, (4.1) holds and

$$
\int_{0}^{t} I_{\{0\}}(x(s)) d \psi(s)=\psi(t)^{6)} .
$$

Later we shall see that $(\mathrm{E})$ implies $\psi(t) \equiv 0$. It is well known that if (4.1) has a unique solution $(x(t), \psi(t))$, then $(x(t), P)$ is a diffusion process corresponding to the generator $L$ of (1.2) with the domain $D(L)$ of (2.6).

The next lemma is a variation of [13] and [19].

Lemma 3. There is a solution $(x(t), \psi(t))$ of (4.1), which is pathwise unique.

Proof. Step 1 (Uniqueness). Suppose that (4.1) has two solutions $\left(x^{(1)}(t)\right.$, $\left.\psi^{(1)}(t)\right)$ and $\left(x^{(2)}(t), \psi^{(2)}(t)\right)$. Choose a sequence $\left\{x_{n}\right\}$ decreasing to 0 and continuous nonnegative functions $g_{n}(x), n=1,2, \ldots$, such that support $\left[g_{n}(x)\right]$ $\subset\left(x_{n}, x_{n-1}\right)$ and $\int_{0}^{\infty} g_{n}(x) d x=1$. Let $f_{n}(x)=\int_{0}^{|x|} \int_{0}^{y} g_{n}(z) d z d y$. Then we have

$$
\begin{aligned}
& E\left[f_{n}\left(x^{(1)}(t)-x^{(2)}(t)\right)\right] \\
& =E\left[\int_{0}^{t} f_{n}^{\prime}\left(x^{(1)}(s)-x^{(2)}(s)\right)\left(b\left(x^{(1)}(s)\right)-b\left(x^{(2)}(s)\right)\right) d s\right] \\
& \quad+E\left[\int_{0}^{t} f_{n}^{\prime}\left(x^{(1)}(s)-x^{(2)}(s)\right) d\left(\psi^{(1)}(s)-\psi^{(2)}(s)\right)\right]
\end{aligned}
$$

(e.g. [7]). Now since $b(x)$ is nonincreasing and (4.2) holds, we see that

$$
x^{(1)}(s)>x^{(2)}(s) \geqq 0
$$

implies

$$
\begin{aligned}
& f_{n}^{\prime}\left(x^{(1)}(s)-x^{(2)}(s)\right) \geqq 0 \\
& b\left(x^{(1)}(s)\right)-b\left(x^{(2)}(s)\right) \leqq 0 \\
& \left(\psi^{(1)}-\psi^{(2)}\right)(s+\varepsilon)-\left(\psi^{(1)}-\psi^{(2)}\right)(s-\varepsilon)=-\left(\psi^{(2)}(s+\varepsilon)-\psi^{(2)}(s-\varepsilon)\right) \leqq 0
\end{aligned}
$$

5) We assume that $\mathscr{F}_{t}$ contains all $P$-null sets, and the assertions in this section should be read to hold almost surely $(P)$.

6) $I_{\Delta}(x)$ is the indicator function of a set $A$. 
for some $\varepsilon>0$. Similarly, $0 \leqq x^{(1)}(s)<x^{(2)}(s)$ implies the opposite inequalities. Hence $E\left[f_{n}\left(x^{(1)}(t)-x^{(2)}(t)\right)\right] \leqq 0$. In view of $f_{n}(x) \uparrow|x|$ as $n \uparrow \infty$, we obtain $E\left[\left|x^{(1)}(t)-x^{(2)}(t)\right|\right]=0$. Noting that $x^{(i)}(t)$ is continuous, we have $x^{(1)}(t)$ $\equiv x^{(2)}(t)$ as well as $\psi^{(1)}(t) \equiv \psi^{(2)}(t)$.

Step 2 (Existence in the case of $(\mathrm{E})$ ). Let $\left\{x_{n}\right\}$ be a sequence decreasing to 0 and let $b^{(n)}(x)=b\left(x \vee x_{n}\right)^{7)}$ for $x \in(-\infty, \infty)$. Since $b^{(n)}(x)$ is bounded and continuous, the equation

$$
x^{(n)}(t)=x_{0}+\beta(t)+\int_{0}^{t} b^{(n)}\left(x^{(n)}(s)\right) d s
$$

has a solution ([14] pp. 76-77). This is unique by the same reason as in Step 1. Now let $\sigma_{x}^{(n)}=\inf \left\{t>0: x^{(n)}(t) \leqq x\right\}$. Then, by the uniqueness

$$
x^{(m)}(t)=x^{(n)}(t), \quad t \leqq \sigma_{x_{n}}^{(n)}, \quad m \geqq n .
$$

Hence $\sigma_{x_{n}}^{(n)}=\sigma_{x_{n}}^{(m)} \leqq \sigma_{x_{m}}^{(m)}$. Letting $\sigma=\lim _{n \rightarrow \infty} \sigma_{x_{n}}^{(n)}$, we can define the inductive limit $\{x(t): t<\sigma\}$ of the processes $\left\{x^{(n)}(t): t<\sigma_{x_{n}}^{(n)}\right\}$. But $\sigma=\infty$, since $g\left(x_{0}\right)$ $\equiv E\left[e^{-\mu \sigma}\right](\mu>0)$ is a solution of

$$
\frac{1}{2} g^{\prime \prime}(x)+b(x) g^{\prime}(x)=\mu g(x), \quad 0 \leqq g(x) \leqq 1,
$$

and such a solution identically vanishes by virtue of $(E)$. The process $x(t)$ is a solution of (4.1) with $\psi(t) \equiv 0$.

Step 3 (Existence in the case of $(\mathrm{R})$ ). Let $\left\{x_{n}\right\}$ be a sequence decreasing to 0 and let $\gamma^{(n)}(x), n=1,2, \ldots$, be nonnegative continuous functions such that $\gamma^{(n)}(x)=1 / x\left(0<x \leqq x_{n+1}\right), \leqq 1 / x\left(x_{n+1} \leqq x \leqq x_{n}\right),=0\left(x_{n} \leqq x\right)$ and $b(x)+\gamma^{(n)}(x)$ is nonincreasing. Then, since the function $b(x)+\gamma^{(n)}(x)$ satisfies $(2.1)-(2.4)$ as well as (E), the equation

$$
x^{(n)}(t)=x_{0}+\beta(t)+\int_{0}^{t} b\left(x^{(n)}(s)\right) d s+\int_{0}^{t} \gamma^{(n)}\left(x^{(n)}(s)\right) d s
$$

admits a unique solution $x^{(n)}(t)$ by Step 2. Further, noting $b(x)+\gamma^{(n)}(x) \geqq$ $b(x)+\gamma^{(n+1)}(x)$, we see that $x^{(n)}(t) \geqq x^{(n+1)}(t), t \geqq 0$, by the standard comparison theorem ([6], [18]). Hence there exists a nonnegative limit $x(t)=\lim _{n \rightarrow \infty} x^{(n)}(t)$. Since $b(x)$ is nonincreasing and $\int_{0}^{t} b\left(x^{(n)}(s)\right) d s \leqq x^{(n)}(t)-x_{0}-\beta(t)$ by $(4.3)$, it follows that

$$
\lim _{n \rightarrow \infty} \int_{0}^{t} b\left(x^{(n)}(s)\right) d s=\int_{0}^{t} b(x(s)) d s, \quad \imath \geqq 0,
$$

7) $a \vee b=\max \{a, b\}$ and $a \wedge b=\min \{a, b\}$. 
the both sides being finite. Now (4.3) and (4.4) imply existence of a finite limit

$$
\psi(t)=\lim _{n \rightarrow \infty} \int_{0}^{t} \gamma^{(n)}\left(x^{(n)}(s)\right) d s, \quad t \geqq 0 .
$$

Hence $(x(t), \psi(t))$ satisfies (4.1).

It is clear that $x(t) \geqq 0$, and $x(t)$ and $\psi(t)$ are $\mathscr{F}_{t}$-adapted. We shall show they are continuous. Since $x(t)$ is nonnegative and upper semicontinuous, it is continuous at the time $t$ for which $x(t)=0$. Let $x\left(t_{0}\right)>0$. Take an $N>0$ such that $x\left(t_{0}\right) \geqq x_{N}$. We can find a $\delta>0$ such that

$$
x^{(n)}(t) \geqq x_{N+1}, \quad t \in\left(t_{0}-\delta, t_{0}+\delta\right) \cap[0, \infty), \quad n \geqq N+1 .
$$

If otherwise, then, by the continuity of $x^{(n)}(t)$, there is a sequence $\left\{t_{n}\right\}$ such that $t_{n} \rightarrow t_{0}, t_{n} \geqq 0, x^{(n)}\left(t_{n}\right)=x_{N+1}$ and $x^{(n)}(t) \geqq x_{N+1}$ for all $t \in\left[t_{0} \wedge t_{n}, t_{0} \vee t_{n}\right]$. But (4.3) gives

$$
\begin{aligned}
0<x_{N}-x_{N+1} & \leqq x^{(n)}\left(t_{0}\right)-x^{(n)}\left(t_{n}\right) \\
& \leqq\left|\beta\left(t_{0}\right)-\beta\left(t_{n}\right)\right|+b\left(x_{N+1}\right)\left|t_{0}-t_{n}\right|, \quad n \geqq N+1,
\end{aligned}
$$

which is impossible. Hence, there exists a $\delta$ satisfying (4.5). Also, equicontinuity of $\left\{x^{(n)}(t): n \geqq N+1\right\}$ on $\left(t_{0}-\delta, t_{0}+\delta\right) \cap[0, \infty)$ follows from (4.3) and (4.5). Hence $x(t)$ is continuous at $t_{0}$. Therefore, $x(t)$ is continuous on $[0, \infty)$. By (4.1), this assures the continuity of $\psi(t)$.

Finally we shall show (4.2). By (4.5) it follows that

$$
\int_{\left(t_{0}-\delta\right) \vee 0}^{t_{0}+\delta} \gamma^{(n)}\left(x^{(n)}(s)\right) d s=0, \quad n \geqq N+1 .
$$

Hence $\psi\left(t_{0}+\delta\right)-\psi\left(\left(t_{0}-\delta\right) \vee 0\right)=0$. Therefore, for each $N, \int_{0}^{t} I_{\left[x_{N}, \infty\right)}(x(s)) d \psi(s)$ $=0$. Letting $N \rightarrow \infty$, we have (4.2).

Lemma 4. 1) Suppose that, for $i=1,2, b_{i}(x)$ satisfies (2.1)-(2.4), and $\left(x_{i}(t), \psi_{i}(t)\right)$ is the solution of (4.1) with $b(x)$ and $x_{0}$ replaced by $b_{i}(x)$ and $x_{0}^{(i)}$. If $x_{0}^{(1)} \leqq x_{0}^{(2)}$ and $b_{1}(x) \leqq b_{2}(x)$, then $x_{1}(t) \leqq x_{2}(t), t \geqq 0$.

2) Let $(x(t), \psi(t))$ be the solution of (4.1) and $(\bar{x}(t), \bar{\psi}(t))$ be that of (4.1) with $\bar{b}(x)=b(x)-h$ and $\bar{x}_{0}$ in place of $b(x)$ and $x_{0}$. If $0 \leqq h<b_{0}$ and $\bar{x}_{0} \leqq x_{0}$, then

$$
x(t)-h t-x_{0} \leqq \bar{x}(t)-\bar{x}_{0}, \quad t \geqq 0 .
$$

Proof. 1) Case 1. When both $b_{1}(x)$ and $b_{2}(x)$ satisfy (E), the assertion is none other than the standard comparison theorem ([6], [18]). 
Case 2. Suppose that $b_{1}(x)$ satisfies $(\mathrm{R})$ and $b_{2}(x)$ satisfies (E). Let $\left\{x_{n}\right\}$ be a sequence decreasing to 0 and let $\tilde{\gamma}^{(n)}(x), n=1,2, \ldots$, be nonnegative continuous functions on $(0, \infty)$ such that $\tilde{\gamma}^{(n)}(x)=b_{2}(x)-b_{1}(x)\left(0<x \leqq x_{n+1}\right)$, $\leqq b_{2}(x)-b_{1}(x)\left(x_{n+1}<x<x_{n}\right),=0\left(x_{n} \leqq x\right)$ and $b_{1}(x)+\tilde{\gamma}^{(n)}(x)$ is nonincreasing. Then the function $b_{1}(x)+\tilde{\gamma}^{(n)}(x)$ can play the role of $b(x)+\gamma^{(n)}(x)$ in Step 3 of the proof of Lemma 3. The corresponding solution $x_{1}^{(n)}(t)$ satisfies $x_{1}^{(n)}(t)$ $\leqq x_{2}(t), t \geqq 0$, by the result of Case 1 . Since $x_{1}^{(n)}(t)$ tends to $x_{1}(t)$, this gives $x_{1}(t) \leqq x_{2}(t), t \geqq 0$.

Case 3. When both $b_{1}(x)$ and $b_{2}(x)$ satisfy $(\mathrm{R})$, we use the $\gamma^{(n)}(x)$ in the proof of Lemma 3. By the result of Case 1, the corresponding solutions satisfy $x_{1}^{(n)}(t) \leqq x_{2}^{(n)}(t), t \geqq 0 . \quad$ This gives $x_{1}(t) \leqq x_{2}(t)$.

2) Suppose that $b(x)$ satisfies $(\mathrm{E})$. Then $\psi(t) \equiv 0$. Since $\bar{x}(t) \leqq x(t), t \geqq 0$, and $b(x)$ is nonincreasing,

$$
\begin{aligned}
x(t)-h t-x_{0} & =\beta(t)+\int_{0}^{t}(b(x(s))-h) d s \\
& \leqq \beta(t)+\int_{0}^{t}(b(\bar{x}(s))-h) d s=\bar{x}(t)-\bar{x}_{0} .
\end{aligned}
$$

In the case that $b(x)$ satisfies $(\mathrm{R})$, let $x^{(n)}(t)$ be the solution of (4.3) and $\bar{x}^{(n)}(t)$ be that of (4.3) corresponding to $\bar{b}(x)$ and $\bar{x}_{0}$. Then $\bar{x}^{(n)}(t) \leqq x^{(n)}(t)$, and

$$
\begin{aligned}
& x^{(n)}(t)-h t-x_{0} \\
& \quad=\beta(t)+\int_{0}^{t}\left(b\left(x^{(n)}(s)\right)-h\right) d s+\int_{0}^{t} \gamma^{(n)}\left(x^{(n)}(s)\right) d s \\
& \quad \leqq \beta(t)+\int_{0}^{t}\left(\bar{b}\left(\bar{x}^{(n)}(s)\right)+\gamma^{(n)}\left(\bar{x}^{(n)}(s)\right)\right) d s=\bar{x}^{(n)}(t)-\bar{x}_{0} .
\end{aligned}
$$

We get the conclusion by letting $n \rightarrow \infty$.

\section{$\S 5$. Proof of Theorem 1}

Using the $\varphi_{\lambda}(x)$ given in Lemma 1 , we define

$$
W^{(\lambda)}(t)=e^{(\lambda-c) t} \check{\varphi}_{\lambda}\left(x_{t}\right)
$$

Then this is a martingale $([17])$. Further, if $0 \leqq \lambda \leqq \lambda_{0}$, then this is a nonnegative martingale, so that there is a nonnegative integrable limit

$$
W^{(\lambda)}=\lim _{t \rightarrow \infty} W^{(\lambda)}(t), \quad \text { a.s. }
$$

Lemma 5. Let $g \in \boldsymbol{B}$ have a compact support. Then, 


$$
\begin{array}{lll}
\lim _{t \rightarrow \infty} e^{(\lambda-c) t} \grave{g}\left(x_{t}\right)=0, & \text { a.s. } & \text { for } \lambda<\lambda_{0}, \\
\lim _{t \rightarrow \infty} e^{\left(\lambda_{0}-c\right) t} \check{g}\left(x_{t}\right)=0, & \text { in } L^{1}(\Omega) .
\end{array}
$$

Proof. Suppose $\lambda<\lambda_{0}$. Using Lemma 1, we can easily show that there is a positive constant $K_{1}$ such that $|g(x)| \leqq K_{1} \varphi_{\lambda_{0}}(x)$. Hence,

$$
\varlimsup_{t \rightarrow \infty} e^{(\lambda-c) t}\left|\check{g}\left(\boldsymbol{x}_{t}\right)\right| \leqq K_{1} \lim _{t \rightarrow \infty} e^{\left(\lambda-\lambda_{0}\right) t} e^{\left(\lambda_{0}-c\right) t} \check{\varphi}_{\lambda_{0}}\left(\boldsymbol{x}_{t}\right)=0, \quad \text { a.s., }
$$

which completes the proof of (5.2).

We shall show (5.3). Without loss of generality, we may assume that $g(x)$ is nonnegative and nonincreasing. By [17],

$$
\boldsymbol{E}_{x}\left[e^{\left(\lambda_{0}-c\right) t} \check{g}\left(x_{t}\right)\right]=e^{\lambda_{0} t} E_{x}\left[g\left(x_{t}\right)\right] .
$$

Let $X^{*}=\left(x_{t}^{*}, P_{x}^{*}\right)$ be a diffusion process given in Section 2 with $b(x) \equiv b_{0}$, and denote the corresponding objects by $p^{*}(t, x, y), T_{t}^{*}$ etc. Then we have $E_{x}\left[g\left(x_{t}\right)\right] \leqq E_{x}^{*}\left[g\left(x_{t}^{*}\right)\right]$ by Lemma 4 . Hence, it is enough to show that

$$
\lim _{t \rightarrow \infty} e^{\lambda_{0} t} T_{t}^{*} g(x)=0 .
$$

It is easily seen that the transition density $p^{*}(t, x, y)$ of $X^{*}$ is represented as

$$
p^{*}(t, x, y)=\int_{\lambda_{0}}^{\infty} e^{-\lambda t} \varphi_{\lambda}^{*}(x) \varphi_{\lambda}^{*}(y) \sigma^{*}(d \lambda), \quad x, y \in S, t>0
$$

where

$$
\begin{aligned}
& \varphi_{\lambda}^{*}(x)=e^{-b_{0} x}\left\{\cos \sqrt{2 \lambda-b_{0}^{2}} x+\frac{b_{0}}{\sqrt{2 \lambda-b_{0}^{2}}} \sin \sqrt{2 \lambda-b_{0}^{2}} x\right\} \\
& \sigma^{*}(d \lambda)=\frac{\sqrt{\lambda-\lambda_{0}}}{\sqrt{2} \pi \lambda} d \lambda .
\end{aligned}
$$

Hence, using Fubini's theorem, we obtain

$$
e^{\lambda_{0} t} T_{t}^{*} g(x)=\int_{\lambda_{0}}^{\infty} e^{\left(\lambda_{0}-\lambda\right) t} \varphi_{\lambda}^{*}(x) \mathscr{F} g(\lambda) \sigma^{*}(d \lambda),
$$

where

$$
\mathscr{F} g(\lambda)=\int_{0}^{\infty} g(x) \varphi_{\lambda}^{*}(x) m^{*}(d x) .
$$

Now for $t \geqq t_{0}>0$ and $\lambda \geqq \lambda_{0}$,

$$
\left|e^{\left(\lambda_{0}-\lambda\right) t} \varphi_{\lambda}^{*}(x) \mathscr{F} g(x)\right| \leqq e^{-\left(\lambda-\lambda_{0}\right) t_{0}} \mathscr{F} g\left(\lambda_{0}\right),
$$

and

$$
\int_{\lambda_{0}}^{\infty} e^{-\lambda t_{0}} \sigma^{*}(d \lambda)=p^{*}\left(t_{0}, 0,0\right)<\infty
$$


Therefore, using Lebesgue's dominated convergence theorem, we have (5.4).

Proof of Theorem 1. 1) Take a $g \in \boldsymbol{B}_{0}\left(\alpha_{\lambda}, l\right)$, and let

$$
\begin{aligned}
& g_{1}(x: R)=\left(g(x)-\varphi_{\lambda}(x) l / l\left(\varphi_{\lambda}\right)\right) I_{[0, R)}(x), \\
& g_{2}(x: R)=\left(g(x)-\varphi_{\lambda}(x) l / l\left(\varphi_{\lambda}\right)\right) I_{[R, \infty)}(x),
\end{aligned}
$$

for each $R>0$. Then we have

$$
g(x)=\varphi_{\lambda}(x) l / l\left(\varphi_{\lambda}\right)+g_{1}(x: R)+g_{2}(x: R) .
$$

Using (3.2), we can easily show that for each $\varepsilon_{n}>0$ there exists a positive $R_{n}$ such that $\left|g_{2}\left(x: R_{n}\right)\right| \leqq \varepsilon_{n} \varphi_{\lambda}(x)$. Hence,

$$
e^{(\lambda-c) t}\left|\check{g}_{2}\left(x_{t}: R_{n}\right)\right| \leqq \varepsilon_{n} W^{(\lambda)}(t) .
$$

Further, since $g_{1}\left(x: R_{n}\right)$ has a compact support,

$$
\lim _{t \rightarrow \infty} e^{(\lambda-c) t} \check{g}_{1}\left(x_{t}: R_{n}\right)=0, \quad \text { a.S., }
$$

by Lemma 5. Hence (5.5) implies

$$
\begin{aligned}
\varlimsup_{t \rightarrow \infty}\left|e^{(\lambda-c) t} \check{g}\left(x_{t}\right)-W^{(\lambda)} l / l\left(\varphi_{\lambda}\right)\right| \leqq & \varlimsup_{t \rightarrow \infty}\left|\left(W^{(\lambda)}(t)-W^{(\lambda)}\right) l\right| l\left(\varphi_{\lambda}\right) \mid \\
& +\varepsilon_{n} \lim _{t \rightarrow \infty} W^{(\lambda)}(t)=\varepsilon_{n} W^{(\lambda)}, n=1,2, \ldots, \quad \text { a.s. }
\end{aligned}
$$

Now let $\varepsilon_{n} \downarrow 0$ and set $W_{\lambda}=W^{(\lambda)} / l\left(\varphi_{\lambda}\right)$. Then (2.8) follows.

2) (2.9) will be proved by a similar way. But the conclusion holds in probability since the assertion in (5.3) holds only in $L^{1}(\Omega)$.

q.e.d.

\section{§6. Proof of Theorem 2}

For the $W^{(\lambda)}(t)$ and $W^{(\lambda)}$ in the previous section, we define

$$
\begin{aligned}
& \psi_{t}(x, a)=\boldsymbol{E}_{x}\left[\exp \left\{-a W^{(\lambda)}(t)\right\}\right], \\
& \psi(x, a)=\boldsymbol{E}_{x}\left[\exp \left\{-a W^{(\lambda)}\right\}\right], \quad a \geqq 0 .
\end{aligned}
$$

Denote $T_{\varepsilon}^{0}=e^{-c t} T_{\varepsilon}$.

Lemma 6. Let $0 \leqq \lambda \leqq \lambda_{0}$. Then

$$
\psi_{i}(x, a)=T_{i}^{0} f_{t}(\cdot, a)(x)+c \int_{0}^{t} T_{s}^{0}\left\{\psi_{t-s}\left(\cdot, a e^{(\lambda-c) s}\right)^{2}\right\}(x) d s,
$$

where $f_{t}(x, a)=\exp \left\{-a e^{(\lambda-c) t} \varphi_{\lambda}(x)\right\}$. Further, the function $v(x) \equiv \mathbb{P}_{x}\left(W^{(\lambda)}\right.$ 
$=0)$ satisfies (3.20) with some $v_{0}$.

Proof. For a fixed $t_{0} \geqq 0, \psi_{t}\left(x, a: t_{0}\right) \equiv \boldsymbol{E}_{x}\left[\hat{f}_{t_{0}}\left(\boldsymbol{x}_{t}, a\right)\right]$ satisfies the $S$-equation (2.7) with $g(x)=f_{t_{0}}(x, a)$. Hence

$$
\psi_{t}\left(x, a: t_{0}\right)=T_{t}^{0} f_{t_{0}}(\cdot, a)(x)+c \int_{0}^{t} T_{s}^{0}\left\{\boldsymbol{E} \cdot\left[\hat{f}_{t_{0}}\left(\boldsymbol{x}_{t-s}, a\right)\right]^{2}\right\}(x) d s .
$$

Since $\psi_{t}(x, a)=\psi_{t}(x, a: t)$ and $\boldsymbol{E}_{x}\left[\hat{f}_{t}\left(\boldsymbol{x}_{t-s}, a\right)\right]=\psi_{t-s}\left(x, a e^{(\lambda-c) s}\right)$, we obtain (6.1).

To prove the second assertion, note that $\lim _{t \rightarrow \infty} \psi_{t}(x, a)=\psi(x, a)$ and

$$
\left\|T_{t}^{0}\right\| \leqq e^{-c t}, \quad\left\|f_{t}(\cdot, a)\right\| \leqq 1, \quad\left\|\psi_{s}(\cdot, a)\right\| \leqq 1,
$$

where $\|f(\cdot)\|=\sup _{x \in S}|f(x)|$ and $\|T\|=\sup _{\|f\|=1}\|T f\|$. Then by letting $t \rightarrow \infty$ in (6.1), we have

$$
\psi(x, a)=c \int_{0}^{\infty} T_{s}^{0}\left\{\psi\left(\cdot, a e^{(\lambda-c) s}\right)^{2}\right\}(x) d s .
$$

Let $a \rightarrow \infty$. It follows that $v(x) \equiv \lim _{a \rightarrow \infty} \psi(x, a)$ satisfies

$$
v(x)=c \int_{0}^{\infty} T_{s}^{0}\left\{v(\cdot)^{2}\right\}(x) d s .
$$

This completes the proof.

Lemma 7. If $0<c \leqq \lambda_{0}$, then

$$
\boldsymbol{P}_{x}\left(W_{c}=0\right)=0, \quad x \in S .
$$

Proof. Fix $0<v_{0}<1$, and let $v(x)$ be the solution of (3.20). Then $u(t, x)$ $\equiv v(x)$ satisfies

$$
u(t, x)=T_{t}^{0} v(x)+c \int_{0}^{t} T_{s}^{0}\left\{u(t-s, \cdot)^{2}\right\}(x) d s .
$$

On the other hand, setting $\lambda=c$ in (6.1), we have

$$
\psi_{t}(x, a)=T_{t}^{0} f(\cdot, a)(x)+c \int_{0}^{t} T_{s}^{0}\left\{\psi_{t-s}(\cdot, a)^{2}\right\}(x) d s
$$

where $f(x, a)=\exp \left\{-a \varphi_{c}(x)\right\}$. But Lemmas 1 and 2 ensure that, for any sufficiently large $a$,

$$
v(x) \geqq f(x, a), \quad x \in S .
$$

Hence, it follows from (6.4) and (6.5) that

$$
\psi_{t}(x, a) \leqq u(t, x)=v(x), \quad x \in S, \quad t \geqq 0 .
$$


Now let $t \rightarrow \infty$ and then $a \rightarrow \infty$. We obtain $\psi(x) \equiv \lim _{a \rightarrow \infty} \psi(x, a) \leqq v(x), x \in S$. Hence, using Lemmas 2 and 6, we complete the proof, because $0<v_{0}<1$ is arbitrary.

Now let us make a convenient realization of the BDP $\boldsymbol{X}$. Let $\beta_{i}(t), i=1$, $2, \ldots$, be one-dimensional Brownian motions, and $\tau_{i}, i=1,2, \ldots$, be random variables with the exponential distribution of mean $1 / c$ on a common probability space $(\Omega, \mathscr{F}, \boldsymbol{P})$. Further suppose that $\left\{\beta_{i}(t), \tau_{j}: i, j=1,2, \ldots\right\}$ are mutually independent. Let also $x_{i}\left(t: x_{0}\right)$ be the solution of (4.1) with $\beta_{i}(t)$ in place of $\beta(t)$. We shall define $\boldsymbol{x}^{e}(t)=\left(x^{1}(t), x^{2}(t), \ldots, x^{\xi^{5}}(t)\right)$ inductively. First, define

$$
\xi_{t}=1, \quad x^{1}(t)=x_{1}(t: x), \quad 0 \leqq t<T_{1},
$$

where $T_{1}=\tau_{1}$. Then define

$$
\begin{aligned}
& \xi_{t}=2, \\
& x^{1}(t)=x_{2}\left(t-T_{1}: x^{1}\left(T_{1}-\right)\right), \quad x^{2}(t)=x_{3}\left(t-T_{1}: x^{1}\left(T_{1}-\right)\right), \quad T_{1} \leqq t<T_{2}{ }^{8},
\end{aligned}
$$

where $T_{2}=\min \left\{\tau_{2}+T_{1}, \tau_{3}+T_{1}\right\}$. Suppose that we have obtained

$$
\begin{aligned}
& \xi_{t}=n, \\
& x^{i}(t)=x_{k_{i}}\left(t-T_{n_{i}}: x^{l_{i}}\left(T_{n_{i}}-\right)\right), \quad 1 \leqq i \leqq n, \quad T_{n-1} \leqq t<T_{n},
\end{aligned}
$$

where $k_{i}, n_{i}$, and $l_{i}$ are some integers satisfying $1 \leqq n_{i} \leqq n-1,1 \leqq l_{i} \leqq n_{i}, T_{n}=$ $\min \left\{\tau_{k_{i}}+T_{n_{i}}: 1 \leqq i \leqq n\right\}$, and $k_{i} \neq k_{j}$ for $i \neq j$. If $T_{n}=\tau_{k_{i_{0}}}+T_{n_{i_{0}}}{ }^{9)}$, then we define, as the next step,

$$
\begin{aligned}
\xi_{t} & =n+1, \\
x^{i}(t) & =x_{k_{i}}\left(t-T_{n_{i}}: x^{l_{i}}\left(T_{n_{i}}-\right)\right), \\
x^{i_{0}}(t) & =x_{2 n}\left(t-T_{n}: x^{i_{0}}\left(T_{n}-\right)\right), \\
x^{n+1}(t) & =x_{2 n+1}\left(t-T_{n}: x^{i_{0}}\left(T_{n}-\right)\right),
\end{aligned}
$$

where $T_{n+1}=\min \left\{\tau_{k_{i}}+T_{n_{i}}: i \neq i_{0}, 1 \leqq i \leqq n\right\} \wedge\left(\tau_{2 n}+T_{n}\right) \wedge\left(\tau_{2 n+1}+T_{n}\right)$. Since $T_{n}$ is increasing in $n$ and $\boldsymbol{P}\left(T_{n} \leqq t\right)=\left(1-e^{-c t}\right)^{n}, \lim _{n \rightarrow \infty} T_{n}=\infty$ a.s. Thus we can define $\boldsymbol{x}^{e}(t)$ for all $t \geqq 0$. Finally denote by $\boldsymbol{x}_{t}$ the equivalent class containing $x^{e}(t)$ in the $n$-fold $\left(n=\xi_{t}\right)$ symmetric product space $S^{n}$ of $S$. Then the process $\left(\boldsymbol{x}_{t}, \boldsymbol{P}\right)$ is a realization of the BDP $\boldsymbol{X}([5],[12]) . \quad\left\{T_{n}\right\}$ is the sequence of splitting times and $\xi_{t}$ is the number of particles at time $t$.

Let $\bar{b}(x)=b(x)-h\left(0 \leqq h<b_{0}\right)$, and $\bar{x}_{i}\left(t: x_{0}\right)$ be the solution of (4.1) with

8) The probability that $x^{i}\left(T_{j}-\right)=0$ is equal to zero. So we can exclude such a case.

9) The probability that more than two $\tau_{k_{i}}+T_{n_{i}}$ attain the minimum simultaneously is equal to zero. Hence $i_{0}$ is well-defined. 
$\beta_{i}(t)$ and $\bar{b}(x)$ in place of $\beta(t)$ and $b(x)$, respectively. Then, repeating the above procedure, we can construct on the same probability space a process $\left(\overline{\boldsymbol{x}}_{t}, \boldsymbol{P}\right)$, a realization of the BDP $\overline{\boldsymbol{X}}$ corresponding to the fundamental system $(\bar{X}, c, \pi)$, where $\bar{X}$ is a diffusion process with the generator $\bar{L}=d^{2} / 2 d x^{2}+\bar{b}(x) d / d x$. Note that the splitting times $T_{n}$ are common to $\left(\boldsymbol{x}_{t}, \boldsymbol{P}\right)$ and $\left(\bar{x}_{t}, \boldsymbol{P}\right)$, since the common $\left\{\tau_{i}\right\}$ is used.

Lemma 8. For each $0 \leqq h<b_{0}$,

$$
x^{i}(t)-h t \leqq \bar{x}^{i}(t) \leqq x^{i}(t), \quad t \geqq 0, \quad i=1,2, \ldots, \xi_{t}, \quad \text { a.s. }
$$

Proof. If $0 \leqq t<T_{1}$, then the assertion is clear from (6.6) and Lemma 4. Suppose that the assertion is valid for all $t<T_{n}$. Then

$$
x^{l_{i}}\left(T_{n_{i}}-\right)-h T_{n_{i}} \leqq \bar{x}^{l_{i}}\left(T_{n_{i}}-\right) \leqq x^{l_{i}}\left(T_{n_{i}}-\right)
$$

for all $l_{i}$ and $n_{i}$ in (6.7). Let $t \in\left[T_{n}, T_{n+1}\right]$. By Lemma 4, it follows that

$$
\begin{aligned}
& \bar{x}_{k_{i}}\left(t-T_{n_{i}}: \bar{x}^{l_{i}}\left(T_{n_{i}}-\right)\right) \leqq x_{k_{i}}\left(t-T_{n_{i}}: x^{l_{i}}\left(T_{n_{i}}-\right)\right), \\
& x_{k_{i}}\left(t-T_{n_{i}}: x^{l_{i}}\left(T_{n_{i}}-\right)\right)-h\left(t-T_{n_{i}}\right)-x^{l_{i}}\left(T_{n_{i}}-\right) \\
& \quad \leqq \bar{x}_{k_{i}}\left(t-T_{n_{i}}: \bar{x}^{l_{i}}\left(T_{n_{i}}-\right)\right)-\bar{x}^{l_{i}}\left(T_{n_{i}}-\right), \quad i \neq i_{0}, \quad 1 \leqq i \leqq n .
\end{aligned}
$$

Hence (6.7) and (6.9) give

$$
x^{i}(t)-h t \leqq \bar{x}^{i}(t) \leqq x^{i}(t), \quad i \neq i_{0}, \quad 1 \leqq i \leqq n .
$$

Similar observation applies to $i=i_{0}$ and $n+1$. The proof is complete by induction.

Now we are ready to prove Theorem 2.

Proof of Theorem 2. Case 1. $0<\alpha_{\lambda}^{2} / 2 \leqq c \leqq \lambda<\lambda_{0}$. We may choose $g_{\lambda}(x) \equiv \exp \left\{-\alpha_{\lambda} x\right\}$ as $g$ in Theorem 1. Let $h=(\lambda-c) / \alpha_{\lambda} \geqq 0$. Then $b_{0}-h$ $=\alpha_{\lambda} / 2+c / \alpha_{\lambda}>0$, since $\alpha_{\lambda}$ solves (2.11). Now let $\overline{\boldsymbol{X}}$ be the BDP for this $h$ and use the realizations $\left(\boldsymbol{x}_{t}, \boldsymbol{P}\right)$ and $\left(\overline{\boldsymbol{x}}_{t}, \boldsymbol{P}\right)$ of $\boldsymbol{X}$ and $\overline{\boldsymbol{X}}$, respectively. Then, by Lemma 8,

$$
e^{(\lambda-c) t} \check{g}_{\lambda}\left(\boldsymbol{x}_{t}\right)=\sum_{i=1}^{\xi_{t}} e^{-\alpha_{\lambda}\left(x^{i}(t)-h t\right)} \geqq \check{g}_{\lambda}\left(\bar{x}_{t}\right), \quad t \geqq 0, \quad \text { a.s. }
$$

Denote the quantities for $\overline{\boldsymbol{X}}$ by putting a bar such as $\bar{\lambda}_{0}, \bar{b}_{0}, \bar{\alpha}_{\lambda}$, and $\bar{W}_{\lambda}$. Then, using (2.11) and the inequality $\alpha_{\lambda}^{2} \leqq 2 c$, we have

$$
c \leqq \bar{\lambda}_{0}, \quad \alpha_{\lambda}=\bar{\alpha}_{c} .
$$

Lemma 7 applied to the BDP $\overline{\boldsymbol{X}}$ says that $\boldsymbol{P}\left(\bar{W}_{c}=0\right)=0$. Hence, noting (6.10), we get (2.10). 
Case 2. $0<\lambda<\lambda_{0} \wedge c$. Since $\lambda>\alpha_{\lambda}^{2} / 2$ by Remark $1, \sqrt{2 c} \alpha_{\lambda}-\alpha_{\lambda}^{2} / 2>\alpha_{\lambda}^{2} / 2$. Hence we can choose a $\mu$ satisfying

$$
\alpha_{\lambda}^{2} / 2<\mu<\left(\sqrt{2 c} \alpha_{\lambda}-\alpha_{\lambda}^{2} / 2\right) \wedge \lambda .
$$

For this $\mu$, set $h=(\lambda-\mu) / \alpha_{\lambda}>0$ and let $\overline{\boldsymbol{X}}$ be the corresponding BDP. Then

$$
\bar{b}_{0} \equiv b_{0}-h>0, \quad \mu<\bar{\lambda}_{0}, \quad \alpha_{\lambda}=\bar{\alpha}_{\mu} .
$$

Now take the above realizations $\left(\boldsymbol{x}_{t}, \mathbb{P}\right)$ and $\left(\overline{\boldsymbol{x}}_{t}, \mathbb{P}\right)$ of $\mathbf{X}$ and $\bar{X}$, respectively. Then, by Lemma 8 ,

$$
e^{(\lambda-c) t} \check{g}_{\lambda}\left(x_{t}\right) \geqq e^{(\mu-c)} \check{g}_{\lambda}\left(\bar{x}_{t}\right), \quad \text { a.s. }
$$

Hence it is enough to see $\bar{v}(x) \equiv \boldsymbol{P}\left(\bar{W}^{(\mu)}=0\right)=0$.

First note that, in the present case,

$$
c>\bar{\lambda}_{0} \text {. }
$$

By Lemma 6 applied to the BDP $\overline{\mathbf{X}}$, the function $\bar{v}(x)$ solves (3.20) with $L$ replaced by $\bar{L}$. It follows from Lemma 2 and (6.13) that $\bar{v}(x) \equiv 0$ or $\equiv 1$. So we have only to show

$$
\overline{\boldsymbol{P}}_{x}\left(\bar{W}^{(\mu)}=0\right)<1
$$

By [17],

$$
\begin{aligned}
\overline{\mathbf{E}}_{x}\left[\bar{W}^{(\mu)}(t)^{2}\right] & =e^{2(\mu-c) t} \overline{\boldsymbol{E}}_{x}\left[\bar{\varphi}_{\mu}\left(\overline{\boldsymbol{x}}_{t}\right)^{2}\right] \\
& =e^{2(\mu-c) t} \bar{M}_{t} \bar{\varphi}_{\mu}^{2}(x)+2 c \int_{0}^{t} e^{2(\mu-c) s} \bar{M}_{s} \bar{\varphi}_{\mu}^{2}(x) d s,
\end{aligned}
$$

where $\bar{M}_{t}=e^{c t} \bar{T}_{t}$. By Lemma 1 applied to $\bar{L}$, we have $\bar{\varphi}_{\mu}(x)^{2} \leqq K \bar{\varphi}_{\mu}(x)$ for some $K>0$. Hence, noting $\bar{M}_{t} \bar{\varphi}_{\mu}(x)=e^{(c-\mu) t} \bar{\varphi}_{\mu}(x)$, we have

$$
\bar{E}_{x}\left[\bar{W}^{(\mu)}(t)^{2}\right] \leqq K\left\{e^{(\mu-c) t}+2 c \int_{0}^{t} e^{(\mu-c) s} d s\right\} \bar{\varphi}_{\mu}(x) .
$$

But $\mu<c$ by (6.12) and (6.13). Thus $\bar{W}^{(\mu)}(t)$ is an $L^{2}$-bounded martingale. So

$$
\overline{\boldsymbol{E}}_{x}\left[\bar{W}^{(\mu)}\right]=\lim _{t \rightarrow \infty} \overline{\boldsymbol{E}}_{x}\left[\bar{W}^{(\mu)}(t)\right]=\bar{\varphi}_{\mu}(x)>0 .
$$

This assures (6.14).

Case 3. $\lambda=\lambda_{0}$. Since $\alpha_{\lambda_{0}}^{2} / 2=\lambda_{0}$, it holds that $c \geqq \lambda_{0}$ by the assumption. If $c=\lambda_{0}$, then (2.10) is proved by Lemma 7. If $c>\lambda_{0}$, then the above argument ensures that $W^{\left(\lambda_{0}\right)}(t)$ is an $L^{2}$-bounded martingale and the function $v(x)$ $=\boldsymbol{P}_{x}\left(W^{\left(\lambda_{0}\right)}=0\right)$ must be $\equiv 0$ or $\equiv 1$. Hence, (2.10) follows as above.

Case 4. $\lambda=0$. In this case, we may take the constant function 1 as $g$ in 
Theorem 1. Then $e^{-c t} \breve{1}\left(\boldsymbol{x}_{t}\right)=e^{-c t} \xi_{t}$ is a martingale for the simple branching process. Hence the assertion is obvious ([3] pp. 109-110).

\section{References}

[1] Asmussen, S. and Hering, H., Strong limit theorems for general supercritical branching processes with application to branching diffusions, Z. Wahrscheinlichkeitstheorie, 36 (1976), 195-212.

[2] Dynkin, E. B., Brownian motion in certain symmetric spaces and nonnegative eigenfunctions of the Laplace-Beltrami operator, A.M.S. Translations, Series 2, 72 (1968), 203-228. (Translation).

[ 3 ] Harris, T. E., The Theory of Branching Processes, Springer, 1963.

[4] Helgason, S., Differential Geometry and Symmetric Spaces, Academic Press, 1962.

[5] Ikeda, N., Nagasawa, M. and Watanabe, S., Branching Markov processes, I, II, III, J. Math. Kyoto Univ., 8 (1968), 233-278: 8 (1968), 365-410: 9 (1969), 95-160.

[6] Ikeda, N. and Watanabe, S., A comparison theorem for solutions of stochastic differential equations and its application, Osaka Math. J., 14 (1977), 619-633.

[ 7 ] Itô, K., Stochastic differentials, Appl. Math. Optimization, 1 (1975), 374-381.

[8] Itô, K. and McKean Jr., H. P., Diffusion processes and their sample paths, Springer, 1965.

[9] Kesten, H., Branching Brownian motions with absorption, Stochastic Processes Appl., 7 (1978), 9-47.

[10] McKean, H. P., An upper bound to the spectrum of $\Delta$ on a manifold of negative curvature, J. Diff. Geometry, 4 (1970), 359-366.

[11] _ Application of Brownian motion to the equation of Kolmogorov-PetrovskyPiscunov, Comm. Pure Appl. Math., XXVIII (1975), 323-331.

[12] Nagasawa, M., Branching property of Markov processes, Lecture notes in Math. 258, Séminaire de Probabilités VI Université de Strasbourg, Springer, 1973, 177-198.

[13] Tanaka, H., A construction of one-dimensional Markov processes with reflecting barrier by stochastic differential equations, RIMS Kokyuroku 112, Kyoto Univ., 1971, 120-133 (in Japanese).

[14] Tanaka, H., Tsuchiya, M. and Watanabe, S., Perturbation of drift-type for Lévy processes, J. Math. Kyoto Univ., 14 (1974), 73-92.

[15] Titchmarsh, E. C., Eigenfunction expansions, Part one, Second ed., Oxford, 1962.

[16] Watanabe, S., On the branching process for Brownian particles with absorbing boundary, J. Math. Kyoto Univ., 4 (1965), 385-398.

[17] — Limit theorem for a class of branching processes, Markov processes and potential theory, ed. Chover, Wiley, 1967, 205-232.

[18] Yamada, T., On a comparison theorem for solutions of stochastic differential equations and its application, J. Math. Kyoto Univ., 13 (1973), 497-512.

[19] — On the uniqueness of solutions of stochastic differential equations with reflecting barrier conditions, Lecture notes in Math. 511, Séminaire de Probabilités $X$ Université de Strasbourg, Springer, 1976, 240-244. 